\title{
Conservative Management of Vertebrobasilar Insufficiency: A Systematic Review
}

\author{
Malik M, Rani M, Kumari R, Kaur J and Sharma P
}

\begin{abstract}
Aim: To review the efficacy of the conventional treatment approaches for Conservative Management of Vertebrobasilar Insufficiency. Method: Extensive data mining has been done in treatment of VBI from Pub med, google scholar, research gate that includes pharmacological and physical treatment of VBI. Study selection, data extraction, and assessment of methodogical quality and clinical relevance were performed independently by two reviewers. Results: Pedro scoring has been used to assess various studies which show the use of medical and physical treatment of VBI. Conclusion: This review article concludes that Conventional therapy is effective in improving signs and symptoms of Vertebrobasilar insufficiency.
\end{abstract}

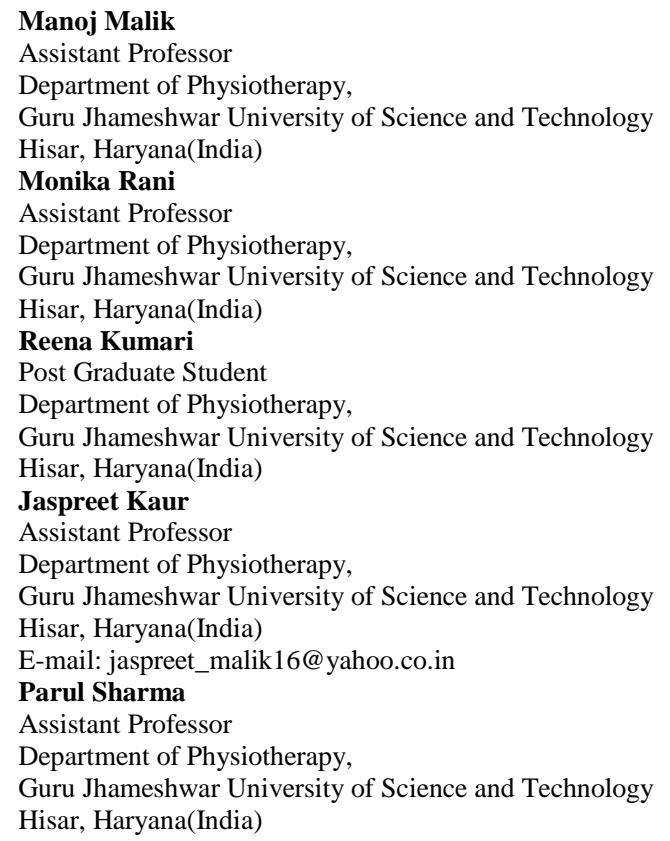

Key Words: Dizziness, Vertigo, Balance, Vestibular Rehabilitation

DOI: $10.18376 / j e s p / 2017 / v 13 / \mathbf{i} 2 / 111283$

\section{Introduction}

Vertebrobasilar insufficiency [VBI] is a condition in which ischemia (blood supply hampered) occurs in vertebral or basilar artery (Jankoski et al, 2005), so it may be considered under intracranial or posterior circulation stroke (Alnaami et al,2012; Caplan ,1980;Cape \& Hogan,1983;Lima Neto et al,2016) according to National Institute of neurological disorder and stroke(NINDS) (Lima Neto et al,2016). Millikan, Siekert, Bradshaw and McQuaid first described the term VBI (Bruyn ,1988) and Kubic and Adams were the person who firstly described its clinical features (Lima Neto et al,2016). It is most commonly seen in men after the fourth decade (Lima 
Neto et al, 2016). It commonly occurs due to injury around the neck especially intervertebral disc, nerve and ligament or muscles (Kerry et al, 2008). According to O'Sullivan and Schmitz occlusion of artery occurs due to cervical rotation and extension movement (Kerry et al,2008) or cerebrovascular accidents (Cape \& Hogan,1983), extracranial lesion, large artery dissection (Alnaami et al, 2012), cervical compressive lesions. The mechanical cause of VBI is fibrous band present at neck, subluxation of apophyseal joint and subaxial spine, osteophyte formation, cervical spondylosis, hypertrophy of uncinate process and facets at cervical spine, stretching and compression of artery and hyperrotation of transverse process (Alnaami I et al, 2012 ; Johnson et al,2008 ; Otto et al, 2008 ; Smith et al,1971 ; Soans\&Khatri, 2014).The less common causes are migraine, fibromuscular dysplasia, coagulopathy and drug abuse (Alnaami et al, 2012 ; Kerry et al,2008 ; Lima Neto et al, 2016 ; Otto et al, 2008 ; Smith et al, 1971 ; Soans\& Khatri,2014] . Most commonly, vertigo is the first sign in VBI patients (Jaspreet et al,2014; Otto et al, 2008). However, in some patients dizziness can be the first complain (Soans\&Khatri, 2014]. Comman's D's means dizzines, drop attack, diplopia, dysarthria and dysphagia, Bilateral sudden hearing loss, Blindness, Diplopia, Dizziness, Drop attacks, Dysarthria, Dysphagia, Dysphagia, Gait ataxia, Headache, Nausea, Nystagmus, Pallor/ tremor can be seen in VBI patients ( APA ,2006; Asavasopon et al,2005; Caplan,1980 ; Cape \& Hogan,1983 ; Johnson et al,2005; Jaspreet et al,2004 ; Kerry et al, 2008 ; Lima Neto et al, 2016 ; Lee.H etal,2003; Namini et al,2015 ; Kidger, 2011 ; Smith et al, 1971 ; Soans\&Khatri, 2014). Most common risk factors of VBI are atherosclerosis commonly due to hypertension and diabeties (Asavasopon et al,2005; Kerry et al, 2008 ; Kidger, 2011 ; Lee H et al, 2003; Namini et al,2015; Cape \& Hogan,1983) and neck pain (Childs et al,2005).

\section{Pathophysiology of VBI}

Thromboembolic VBI usually occurs due to abnormality present in the vertebral or basilar artery which is due to atheromatous plaque formation. Embolus or thrombus formation develops due to trauma in neck which causes atherosclerosis of vertebral artery and vascular accidents. The other pathophysiology of VBI is that, there is thrombosis formation at spinal subclavian trunk which further lead to neurological deficit and causes thromboembolic insufficiency (Cape \&Hogan, 1983). Any injury to head and neck causes release of inflammatory substance which further leads to infection and creating a vicious cycle which starts a cascade of inflammatory event takes place which lead to thromboembolic VBI (Kerry et al, 2008). Hemodynamic VBI occur when there is decrease in blood volume present in the spinal artery which may be result of compression or stenosis of artery due to plaque formation (Maigne, 2006). Lima Neto et al (2016) concluded in a study that atherosclerosis is not the only most common cause of VBI but vestibular system also plays an important role. According to him if there is complexity present in the irrigation pattern of vestibular system or smaller labyrinth artery branches, both will lead to decreased blood supply which result in ischemia of vestibular labyrinth system causing vertigo and VBI. The diagnosis of VBI is done by using Magnetic resonance imaging, Doppler Ultrasound and Digital subtraction cerebral angiography(DSA) and single photon emission computed tomography (SPECT) (Namini et al,2015 ; Johnson et al, 2008 ; Alnaami et al, 2012 ; Maigne et al, 2006) and several tests e.g. De Kleyne's test, Hautant's test (Jaspreet et al, 2004), End range motion test (APA, 2006; Asavasopo et al, 2005; Childs et al, 2005 ; Kerry et al, 2008] provocation test (Asavasopon et al, 2005). The differential diagnosis of VBI is BPPV, cervicogenic dizziness, vestibular neuritis, labyrinthitis hypotension (Asavasopon et al, 2005), inner ear disease (Alnaami et al, 2012), muscle imbalance, trigger point in SCM and upper trapezius (Johnson et al, 2008), trauma, meningitis and ototoxic drugs (Lee, 2003). The conservative treatment of VBI is reduced head movement, antiplatelet drugs alone or along with aspirin (Cape \& Hogan, 1983), antihistamine [dimenhydrinate], anticholinergic [Scopolamine], calcium channel blocker [cinnarizine, flunarizine], histamine analogs [betahistine], diuretics, neuroleptics, corticosteroids, hemorheologics (Otto et al, 2008). If VBI occurs due to 
vertebral compression (Soans\&Khatri, 2014) due to osteophytes then anterior cervical fusion tehnique is done (Smith et al, 1971). The physiotherapy treatment involves integrated manual therapy especially $\mathrm{C} 0-\mathrm{C} 1$ mobilization, suboccipital release, chin tuck exercises, strengthening exercise especially for scapular retractors, diplopia exercises, exercises for relaxation eg Jacobson relaxation technique and vestibular rehabilitation exercise (Soans\&Khatri, 2014), cervical collar mostly for balance impairments (Jaspreet et al,2004), acupuncture (Qi-xue et al, 2008 ; Qiong, 2009) and electrotherapy like traction, hot packs, ultrasound, LASER. These causes normal flow of vertebral artery and reduces the risk of VBI (Jaspreet et al, 2004;Lukowic et al, 2011).

\section{Methodology}

Literature search was done by comprehensive computerized search on Pub med, Google Scholar, Springer link, Research gate. Review was performed according to PRISMA which sets standard in the reporting of systematic reviews and metaanalysis. Step wise flow diagram of PRISMA is shown in figure 2. Our final study group included 9 articles. Selected articles were evaluated by PEDRO SCORE. References of these studies and of earlier reviews were also examined. The main aim of this review was to summarize the results of studies designed to evaluate conservative treatment (medical and physical therapy) of VBI. Full text articles regarding efficacy of conservative management in VBI were included in the present study. Case studies, experimental studies on efficacy of surgical intervention in VBI, review articles, non experimental studies regarding conventional treatment of VBI were excluded.

Search strategy: PubMed, Google Scholar, Springer link, Research gate were sequentially searched to identify relevant studies.

Assessment of study quality: To assess the quality of study, Pedro scale, which is an 11 item checklist shown in table 1 was used.

Data extraction: Study designs, number of participants, participant characteristics and diagnoses, interventions and result were withdrawn.

Result

Search results. Search retrieved 75 articles of which 2 were repeated, 11 were non experimental studies, and 53 were abstracts, case study, review articles, surgical treatment. 9 fulfilled the selection criteria and have been mentioned in table 3.These articles were selected from Google scholar, Pub med, Research gate. Pedro scoring has been done in table 4. The result outcome of all the study is that medical and physical therapy improves symptoms like vertigo, dizziness, headache, visual disturbances etc in VBI.

\section{Discussion}

The purpose of this article was to find the efficacy of conservative treatment which includes both medical and physical therapy in treatment VBI. Nine studies fulfilling selection criteria were selected after extensive data mining. In this review the studies included medical treatment (cinnarizine and dimenhydrinate, betahistine) and physical treatment (acupuncture, LASER therapy, collars etc). In a study in 2008, Otto et al concluded that fixed combination containing $20 \mathrm{mg}$ cinnarizine and $40 \mathrm{mg}$ dimenhydrinate is effective antivertiginous medication in the treatment of patients suffering from vertigo due to VBI. In VBI, visuo-occulomotor reflexes together with cervical test are more pathological than are vestibuloocular reflexes. In a study of 150 patients by Kazmieerezak et al, the symptoms of visuo-occulomotor disorders were corrected more effectively after betahistine treatment than were vestibulooculomotor reaction. Thrice daily $16 \mathrm{mg}$ dose of betahistine was more effective as compared to $8 \mathrm{mg}$ given thrice daily. Spruill et al (1975) conducted a double-blind, randomized, placebo-controlled, crossover study that does not support the use of betahistine hydrochloride as a treatment for decreasing the frequency of TIAs in vertebral-basilar insufficiency. Sidenafil citrate (Viagra) has vasodilatory effects which improves symptoms of VBI. In our knowledge, few studies are available on physiotherapy management of 
VBI. A study was done by kaur et al in 2004 to find out the effect of collar on balance in Vertebrobasilar insufficiency patients. It was hypothesized that wearing collar improves balance in patients with VBI. Cerrahoghu et al have given a treatment program for VBI in which traction, hot packs, ultrasound and exercises were given. They found that vertebral artery flow significantly improved in normal and hyperextension positions after the treatment. A study was done by Huang Qiong (2009) to find out the therapeutic effects of filiform needle acupuncture combined with dermal needle tapping for vertebrobasilar ischemic vertigo and was found effective, of the 50 cases treated, 39 cases $(78 \%)$ were cured, 6 cases $(12 \%)$ relieved, 4 cases $(8 \%)$ improved, and 1 case (2\%) failed, with a total effective rate of $98 \%$. A recent study by Lukowicz (2011) concluded that laser stimulation 10 times over 2 weeks helps in the improvement of global stability, balance and VBI symptoms due to better blood flow velocity. In this review we collected the studies which included the medical and physical therapy treatment of VBI and was found useful and helpful in alleviating signs and symptoms Of VBI. The studies included in this review had maximum use of cinnarizine and dimenhydrinate which is most effective and safe drug in improving symptoms of VBI. Further research should be done on physiotherapy management of VBI as very sparse literature is available regarding efficacy of physiotherapy in management of VBI.

\section{Conclusion}

Most of the studies suggested that symptom of VBI like vertigo, dizziness, visual disturbances, headache, tinnitus etc improved after administration of drug or physical therapy. The present review concluded that conventional therapy is effective in improving signs and symptoms associated with VBI.

Acknowledgement: I hereby acknowledge my co-authors for constantly encouraging me and helping me find my literature for this review article. We also thank department of physiotherapy, GJUS\&T, Hisar for using the assessts so that, the study could run smooth.

\section{References}

Alnaami, I., Siddiqui, M.,Saqqur, M. 2012.The Diagnosis of Vertebrobasilar Insufficiency Using Transcranial Doppler Ultrasound. Case Reports in Medicine. doi:10.1155/2012/894913,1-3.

Asavasopon, S., Jankoski, J., Godges, J.J. 2005. Clinical Diagnosis of Vertebrobasilar insufficiency: Resident's Case problem. Journal of Orthopedics\& Sports Physical Therapy. 35(10):645-650.

Baloh, R.W. 1975. Vertebrobasilar insufficiency and stroke.OTOLARYNGOL HEAD NECK SURG .112(1):114- 117.

Bozgeyik, Z., Berilgen, S., Ozdemir, H., Tekatas, A., Ogur, E.2008. Evaluation of the effects of sildenafil citrate (viagra) on vertebral artery blood flow in patients with vertebrobasilar insufficiency. Korean J Radiol. 9(6): 477-480.

Bruyn, G.W. Vertigo and Vertebrobasilar Insufficiency. 1988: 128-134.

Cape, R.D.T., Hogan, D.B.1983. Vertebral-Basilar Insufficiency. CAN. FAM. PHYSICIAN. 29:305-308.

Caplan, L.R, .1980. Vertebrobasilar disease, time for a new strategy. Stroke .12(1): 111-114.

Childs, J.D., Flynn, T.W., Fritz, J.M., Piva, S.R., Whitman, J.M., Wainner, R.S., Greenman, P.E. 2005. Screening for vertebrobasilar insufficiency in patients with neck pain:Manual Therapy decisionmaking in the presence of uncertainty. Journal of orthopedic and sports physical therapy. 35(5): 300306.

Clinical guidelines for assessing vertebrobasilar insufficiency in management of cervical spine disorders. 2006. Australian Physiotherapy Association.

Jaspreet, K., Manoj, M., Inderpreet, K., and Thakral, N. 2014. Effect of Collar on Balance in Vertebrobasilarinsuffciency Patients. Indian Journal of Physiotherapy and Occupational Therapy. 8 (1):198-203

Johnson, E.G., Appel, T.D., Landel, R., Kusunose, R.S. 2008. Positive patient outcome after manual cervical spine management despite a positive vertebral artery test. Manual Therapy.13: 367-371.

Kazmierczak, H., Osinska, K. P., Kazmierczak, W. 2004. Betahistine in Vertebrobasilar insufficiency. International Tinnitus Journal. 10 (2):191-193. 
Kerry, R., Taylor, A.2006. Cervical arterial dysfunction assessment and manual therapy. Manual Therapy.11:243-253.

Kerry, R., Taylor, A.J., Mitchell, J., McCarthy, C., Brew, J. 2008.Manual Therapy an Cervical Arterial Dysfunction, direction, for Future: A Clinical Perspective. The Journal of Manual and Manipulative.16 (1): 39-48.

Kidger, P.2011. Vertebrobasilar insufficiency. 1-5

Lee, H., Yi H.A., Baloh, R.W.2003. Sudden bilateral simultaneous deafness with vertigo as a sole manifestation of Vertebrobasilar insufficiency. J Neural Neurosurg psychiatry. (74): 539-541.

Lima Neto A.C, Bittar R, Gattas G.S, Bor-Seng-Shu E.S, Oliveira M.L, Monsanto R.C, Bittar L.F. Pathophysiology and Diagnosis of vertebrobasilar insufficiency : A Review of the literature. International archives of otorhinolaryngology.2016

Lukowicz, M., Zalewski, P., Bulatowicz, I., Buszko, K., Klawe, J.J. 2011. The impact of laser irradiation on global stability in patient with Vertebrobasilar insufficiency. Med Sci Monit.7 (9): CR517-522

Maigne, R. Diagnosis and treatment of pain of vertebral origin,second edition.2006;191-193.

Martines, F., Agrifoglio, M., Bentivegna, D., Mucia, M., Salvago, P., Sireci, F., Ballacchino, A. 2012. Treatment of tinnitus and dizziness associated vertebrobasilar insufficiency with a fixed combination of cinnarizine and dimenhydrinate. 28: 291-296.

Namini, A., Naylor, M., Koenigsberg, R.A. 2015.Vertebrobasilar Insufficiency and Stroke- A Review of Posterior Circulation Diagnostic Imaging and Endovascular Treatment Options. J Am Osteopath CollRadiol. 4(3): 15-23.

Otto, V., Fischer, B., Schwarz, M., Baumann, W., Effenberger, R.P. 2008.Treatment of vertebrobasilar insufficiency - Associated Vertigo with a Fixed Combination of Cinnarizine and Dimenhydrinate. International tinnitus journal. 14(1): 57-67.

Pytel, J., Nagy, G., Tóth, A., Spellenberg, S. , Schwarz, M., Répassy, G.2007. Efficacy and tolerability of a fixed low-dose combination of cinnarizine and dimenhydrinate in the treatment of vertigo: A 4week, randomized, double-blind, active- and placebo-controlled, parallel-group, outpatient study. 29(1): 84-98.

Qiong, H.2009. Fifty Cases of Vertebrobasilar Ischemic Vertigo Treated by Acupuncture. Journal of Traditional Chinese Medicine.29 (2):87-89.

Qi-xue, G. E., Yan, W., Chun-ling, W., Shao-jie, W. 2008. Forty-six Cases of Vertebrobasilar Insufficiency Treated by Acupuncture plus Intravenous Infusion of Ligustrazine. Journal of Traditional Chinese Medicine. 28(4): 245-249.

Shah P.S, Kale J.S. A study of the effects of a vestibular rehabilitation program on patients with peripheral vestibular dysfunctions. The Indian journal of Occupational therapy. (36): 11-16.

Shigeta T, Nakagawa T, Takashima T, Yamane H, Nakal Y. 2009.Clnical value of MRA analysis of the Vertebrobasilar system in vertiginous patients. 47-53.

Smith, R.D., Vanderark, G.D., Kempe, L.G.1971. Cervical spondylosis causing vertebrobasilar insufficiency: a surgical treatment. J. Neueol. Neurosurg. Psychiat. 34: 88-392.

Soans, J., Khatri, S.2014. Effectiveness of Integrated Manual Therapy in VertebrobasilarInsufficiency.VIMS Health Science Journal.1 (3): 139-143.

Spruill, J.H., Toole, J.0., Kitto, W., Miller, H.E. 1975. A Comparison of Betahistine Hydrochloride with Placebo for Vertebral-Basilar Insufficiency: A Double-Blind Study. Stroke. 6: 116-122. 